\title{
Affordability of IBM Cognos business intelligence tool features suitable for small-and medium-sized enterprises' decision-making
}

\begin{tabular}{|c|c|}
\hline \multicolumn{2}{|c|}{$\begin{array}{l}\text { Authors: } \\
\text { Tshepo Magoma }{ }^{1} \text { — } \\
\text { Sithembiso Khumalo } \\
\text { Tanya du Plessis }{ }^{1}\end{array}$} \\
\hline \multicolumn{2}{|c|}{$\begin{array}{l}\text { Affiliations: } \\
{ }^{1} \text { Department of Information } \\
\text { and Knowledge } \\
\text { Management, School of } \\
\text { Consumer Intelligence and } \\
\text { Information Systems, College } \\
\text { of Business and Economics, } \\
\text { University of Johannesburg, } \\
\text { Johannesburg, South Africa }\end{array}$} \\
\hline \multicolumn{2}{|c|}{$\begin{array}{l}\text { Corresponding author: } \\
\text { Tanya du Plessis, } \\
\text { tduplessis@uj.ac.za }\end{array}$} \\
\hline \multicolumn{2}{|c|}{$\begin{array}{l}\text { Dates: } \\
\text { Received: } 31 \text { July } 2020 \\
\text { Accepted: } 18 \text { Jan. } 2021 \\
\text { Published: } 29 \text { Mar. } 2021\end{array}$} \\
\hline \multicolumn{2}{|c|}{$\begin{array}{l}\text { How to cite this article: } \\
\text { Magoma, T., Khumalo, S. \& } \\
\text { Du Plessis, T., 2021, } \\
\text { 'Affordability of IBM Cognos } \\
\text { business intelligence tool } \\
\text { features suitable for } \\
\text { small-and medium-sized } \\
\text { enterprises' decision-making', } \\
\text { South African Journal of } \\
\text { Information Management } \\
\text { 23(1), a1291. https://doi. } \\
\text { org/10.4102/sajim.v23i1.1291 }\end{array}$} \\
\hline \multicolumn{2}{|c|}{$\begin{array}{l}\text { Copyright: } \\
\text { ๔ 2021. The Authors. } \\
\text { Licensee: AOSIS. This wo } \\
\text { is licensed under the } \\
\text { Creative Commons } \\
\text { Attribution License. }\end{array}$} \\
\hline \multicolumn{2}{|l|}{ Read online: } \\
\hline 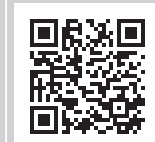 & $\begin{array}{l}\text { Scan this QR } \\
\text { code with your } \\
\text { smart phone or } \\
\text { mobile device } \\
\text { to read online. }\end{array}$ \\
\hline
\end{tabular}

Background: Business intelligence (BI) tools are generally associated with organisations that have resources to purchase and implement these tools. Evidences abound regarding the correlation between BI tools and improved business decision-making. This study's unit of analysis is affordability as a feature of IBM Cognos making it suitable for small-and medium-sized enterprises (SMEs).

Objective: The research aim was to identify the fundamental features of IBM Cognos which would address decision-making needs of SMEs. The objective was to determine the significance of BI tool features by means of identifying affordable features suitable for SMEs' decision-making.

Method: Quantitative research design and a deductive approach were best suited for assessing the fundamental features of IBM Cognos for SMEs' decision-making needs. The signification framework variables, such as presumed, prized and perceived value of BI tool features, were quantified and measured using statistical analysis tools. A non-probability convenience sampling technique was used with a sample size of 200, that is, 80 BI consultants, 60 SME BI developers and 60 SME managers.

Results: Affordable key features of BI tools in the context of SMEs' business decision-making include consistency and comfort, intuitive interface, avoiding impulsivity, cost effectiveness, availability of information, best programmed visualisations, reporting quickly and easily and financial decision-making.

Conclusion: The signification framework's presumed, prized and perceived value indicators link affordable BI tool features to the consistency of the decision-making process and present an alternative view of affordability. An intuitive interface relates to convenience and ease of authoring content, designing, building and securing reports to the SME, which helps in improving consistent decision-making.

Keywords: business intelligence; BI; BI tools; IBM Cognos; small- and medium-sized enterprises (SMEs); decision-making.

\section{Introduction}

The affordability of business intelligence (BI) tools is generally considered in terms of licence cost, hardware, implementation, maintenance, support, training, hardware and hosting fees in the event of cloud-based solutions.

Comparisons regarding different BI tools, such as Tableau, Microsoft BI, Qlik Sense, Oracle OBIEE, QlikView, SQL Server, MicroStrategy and Microsoft SQL Server Reporting Services abound. This study was not aimed at adding yet another comparison of these tools; instead, it addressed the research gap in terms of the significance of affordability as a feature of a specific BI tool, IBM Cognos. In this study, significance is measured by means of comparing the perceived, presumed, predicted, prized and produced value of the tool for small- and mediumsized enterprises (SMEs). In this manner, the study challenges the general definition of affordability by means of applying an information and knowledge management signification framework.

Any organisation, regardless of its size, must understand its internal and external environment if it wants to improve overall business performance (Ehlers et al. 2019). Small- and medium-sized enterprises are characterised as companies that have limited resources and capital and lack 
infrastructure and skills, yet they have an important role in improving the economic position of their country (Mayeng \& Van Vuuren 2017). Small- and medium-sized enterprises face many issues in the South African context such as access to accurate information, right to information packaging, access to appropriate tools and systems vendors and scope of business information (Ehlers et al. 2019; Ladzani \& Van Vuuren 2002). The application of BI tools can assist companies in becoming flexible and efficient when responding to continuously changing conditions of business (BMI Research Technology Report 2018).

Thamir and Poulis (2015) state that the ideal utilisation of BI tools is dependent upon an information technology (IT) team and its support.

By implication, an SME is required to expand their workforce in the IT domain to deal with functions of a BI suite such as IBM Cognos. IBM Cognos is associated with BI techniques to bring about advanced planning, monitoring and data visualisation with predictive analytics that provide great benefits on a single platform. IBM Cognos enables companies to obtain any kind of data or report that may additionally provide comprehensive reporting as well as convenient access to information required in a fast and easy manner. The problem is that SMEs are lagging in the utilisation of BI tools (BMI Research Technology Report 2018; eds. Kousalya, Balakrishnan \& Raj 2017).

To address this problem of SMEs, SME managers require knowledge of BI tools. Roitzsch et al. (2012) state that although the trend of development of BI tools is increasing in decision support of large organisations, SMEs are reluctant to invest in BI tools. A potential cause is that SMEs face challenges in terms of the affordability of IT solutions. For example, some SMEs face budget constraints and low access to credit which means that BI tools are excluded from their budget (BMI Research Technology Report 2018; Mayeng \& Van Vuuren 2017). In this study, the argument is that the significance of BI for SMEs is determined by the affordability of BI tools. Significance is defined as, 'the quality of being worthy of attention; importance, consequence' (OED 2020b). The unit of analysis is affordability as a feature of IBM Cognos assisting SMEs in the process of decision-making.

This study has relevance to SME managers as they will be able to evaluate the significance of a BI tool such as IBM Cognos' features suitable for SME decision-making needs. To this end, the research question is, 'what is the significance of the fundamental features of IBM Cognos BI tools for SME decision-making?' To answer the question, the article begins with a literature review to develop a conceptual framework that links BI and SME decision-making, the affordability of BI tools and significance of IBM Cognos' fundamental features. Then the research methodology section explains the research design and methods, which is followed by analysis and discussion of the findings. The article ends with a conclusion, recommendation, value of research statement and suggestion for future research.

\section{Literature review}

The utilisation of BI tools by SMEs has been the topic of studies in the past. However, such studies have not utilised a signification framework to evaluate the significance of BI tool features. This section of the article presents the theory of the signification framework. The five categories of significance are firstly established by means of a literature review of the presumed relevance of a BI tool in the context of SME business decision-making, the predicted value in terms of SME's business decision-making, the prized indicators of the key features of IBM Cognos, the perceived value as from the perspective of SMEs and the produced value experienced by SMEs.

\section{Small- and medium-sized enterprises and business intelligence tools}

In South Africa, SMEs typically have fewer than 200 employees, an annual turnover of less than R64 million, capital assets of less than R10m and direct managerial involvement by owners (RSA, Act 23 2003). Small- and medium-sized enterprises need to polish their IT skills in order to remain relevant in the 'technology-centred fourth industrial revolution (4IR)' (Mathu 2019:7). Business intelligence is important in the 4IR to 'detect significant events and identify/ monitor business trends to adapt quickly to their changing environment' (Oztemel \& Gursev 2020:158).

Although Nogués and Valladares (2017) proffer low-cost BI solutions to SMEs, they do not mention the 4IR. To distinguish 4IR BI tools, Turi (2020) describes, for example, the IBM Cognos' causal artificial intelligence (AI) features for reliable BI.

Reliable BI processes require modern analytical tools to transfer competitive and internal information to organisational decision-makers (Turi 2020). Business intelligence combines infrastructure, tools, applications and practices that enable a company to access data to improve and optimise business performance through integration, presentation, analysis and collection of information associated with business (Ghazanfari, Jafari \& Rouhani 2011; Turban et al. 2013; Wu et al. 2014). One of the major features of a BI tool is its capability to reflect systems playing a vital role in the process of strategic planning (Kimball et al. 2015). Business intelligence tools allow organisations to store, obtain and analyse data in order to aid decision-making through reliable reporting and analysis (Fan, Lau \& Zhao 2015; Lim, Chen \& Chen 2013).

The above literature categorically recognises within this study's signification framework the significance of BI, which is found in perceived value in terms of decision-making.

\section{Decision-making and IBM Cognos}

Decision-making positively influences organisational productivity (Chng et al. 2015). Various management problems can be analysed by looking at the aspects of decisionmaking (Chng et al. 2015). Aspects of decision-making identify 
choices available among alternatives, explain Eden and Ackermann (2013), and therefore, decision-making is the art of making that choice. Art appreciates and organisations prize BI tools that facilitate the distinct steps at choosing the correct action that is regarded among the vital functions of management of organisations. Managers need tools to manage the performance of organisations, for example, IBM Cognos is a performance management BI software suit. IBM Cognos is a software that is designed to enable business users to obtain data, assemble reports and assess it even without having technical knowledge (Larson \& Chang 2016).

IBM Cognos software products can be incorporated with rational or multidimensional sources of data of several suppliers such as Oracle, SAP, Microsoft and Teradata (Turi 2020). While multinational companies have been able to successfully use such BI tools for optimum business efficiency, some SMEs may experience concerns regarding affordability. Tableau, Oracle, SAP, Microsoft, Teradata and IBM Cognos require ample resources (Batt et al. 2020; Cooney 2020; Richardson et al. 2020; Turi 2020), which by implication means that SMEs are most probably excluded from the prized benefits of BI tools.

\section{Benefits of IBM Cognos business intelligence tools}

There are many useful features of IBM Cognos (Kim et al. 2013). These features let users explore data, customise and assemble data to follow and generate a unique and fresh business perspective (Davis \& Woratschek 2015; Kim et al. 2013). If a company needs to adopt a separate tool, it would be hard to summarise statistical results for primary business reporting (Nogués \& Valladares 2017; Turi 2020). IBM Cognos BI tools offer statistical and fact-based proof to users to support key decisions (Chii et al. 2016). Furthermore, Kim et al. (2013) mention characteristics of advanced calculations for data modules and dashboards. Rusaneanu (2013) identifies characteristics like better streaming in reports and the ability to produce images as the most prized features of IBM Cognos. Horakova and Skalska (2013) identified prized features of BI tools as intuitive boundary for authoring content, data protection, scheduling and alert, contextualised smart search and best automatic visualisations. Oztemel and Gursev (2020) and Turi (2020) add the significance of being able to predict and create scenarios, which is made possible by means of AI for decision-making in the $4 \mathrm{IR}$.

\section{Decision-making needs of small-and medium-sized enterprises and IBM Cognos features}

Artificial intelligence is one of the pillars of the 4IR that qualifies the significance of BI analytical predictive tools, but the cost of AI disqualifies it as a pillar in the process of SMEs' decision-making (European Commission 2019; Turi 2020).
Instead, the consistency of the process of decision-making has greater significance for SMEs - greater than, for instance, AIpowered machine learning capability. Consistency in the process of decision-making is a major need of SMEs (Steinerowska-Streb \& Steiner 2014). In addition to consistency, other elements may play a significant role in SME decision-making, such as comfort, convenience and thoroughness (Antons \& Piller 2015; Ates et al. 2013). Caution is made regarding the element of impulsivity that leads to decisions with negative outcomes (Antons \& Piller 2015; Nogués \& Valladares 2017). Thus, SMEs must avoid impulsive decision-making. Instead, Pettigrew (2009) reiterates the importance of consistency in the process of decision-making.

From the above discussion, the significance of BI tools for SMEs is linked to consistency of the process and affordability of tools. This does not exclude BI tools associated with the 4IR; on the contrary, Llave, Hustad and Olsen (2018:200) suggest a 'gradual investment strategy is preferable for SMEs' to gain from BI tool predictive features. Such a strategy will make the affordability of BI tools possible.

\section{Affordability of business intelligence tools}

Generally, affordability describes a thing of cost that is not too high (Merriam-Webster dictionary 2020); and something that can be afforded and reasonably priced (OED 2020a). Cost has the most significant impact on BI adoption in SMEs (BecerraGodínez et al. 2020). Small- and medium-sized enterprises should consider it as 'a BI project that involves costs, impacts, and sources' (Becerra-Godínez et al. 2020:308), and not view cost as a barrier. For example, Gurjar and Rathore (2013) describe IBM Cognos as affordable because it has helped SMEs to expand conventional horizons of $\mathrm{BI}$ in terms of providing tools for planning, scenario modelling, real-time monitoring and predictive analytics.

The results of a study by IBM (2013) indicate that affordable systems with easy implementation have the potential to provide strong business analytics to companies. Affordable solutions offer reporting, scorecard, dashboard, forecasting and budgeting features (IBM 2013). These features of IBM Cognos can increase SME decision-making capabilities (Gurjar \& Rathore 2013). Loshin (2012) reports that with IBM Cognos, companies can enhance benefits by minimising the cost of organising data and analysis. IBM typically partners with customers to provide solutions to resolve real-world problems of businesses so that the probability of success can be increased, and cost of ownership minimised (IBM 2010). IBM Cognos makes provision for consolidating management systems and features including task-based and proactive management systems to enable IT departments to manage the cost of information effectively (IBM 2012).

It is possible to perform BI system updates in an economic manner (Gurjar \& Rathore 2013). Small- and medium-sized enterprises require affordable BI tools that provide solutions 
such as install, utilise and apply in the company (IBM 2013). Changes in BI tools can be implemented frugally to help in organisational decision-making (Loshin 2012). Some SMEs utilise BI applications to obtain both short-term and longterm data along with production figures (Gangadharan \& Swami 2004). Wagner and Paton (2014) state that BI tools save SMEs' cost of production. Although SMEs are aware that with the use of BI tools they could excel in their business, they fail to enjoy benefits of BI because of a shortage of resources (Roitzsch et al. 2012).

The affordable features of IBM Cognos enable SMEs to achieve relevance in their decision-making process (BecerraGodínez et al. 2020; Canes 2009). For instance, IBM Cognos includes survey features to get product reviews, consumer behaviour, overview of financial spectrum and the ability to make decisions by means of utilising a BI dashboard and automatic visualisations of the data to facilitate the process of decision-making.

Adkison (2013) describes BI dashboards as data visualisation tools displaying the status of business analytics on a screen with key performance indicators. Business intelligence dashboards equip SMEs with advanced calculations, an intuitive boundary for authoring content, data protection, scheduling and alert, contextualised smart search and best automatic visualisations in order to make decisions in a dynamic context.

Dynamic decision-making deals with many strategic paradoxes and requires consistency in order to be effective (Smith 2014). Previously mentioned, consistency in terms of the process of decision-making is one of the major needs of SMEs (Steinerowska-Streb \& Steiner 2014). A decision-making model is a consistent way of making choices; for example, SME must begin by identifying the decision, obtaining relevant data or information before deciding, identifying alternatives out of which one is to be chosen, weighing evidence, choosing among alternatives, and taking required action based on decision, and end with reviewing the decision and consequences (Cascetta et al. 2015; Smith 2014).

The above underpinning of the significance of BI tools for SME decision-making is illustrated in the conceptual framework developed for this study (Figure 1).

The conceptual framework (Figure 1) represents the decisionmaking needs of SMEs linked to the affordable features of IBM Cognos as identified in the study of Magoma (2018). Consistency is one of the major needs of SMEs. To this end, SMEs require reliable decision-making tools that will ensure comfort and convenience, avoid impulsivity, remain cost-effective and allow SMEs to obtain short- and long-term data. Likewise, SMEs require affordable features of IBM Cognos such as an intuitive interface, data protection, scheduling and alert features, best automatic visualisations and advanced calculations (Magoma 2018). The prominent yellow compositions represent significance; the quality of being worthy of attention; importance, and consequence that is signified by something; meaning, import and implication (OED 2020b), which represents this study's signification framework as described in the 'Research design and methods' section.

\section{Research design and methods}

The research design was guided by the research objective, namely to determine the significance of BI tools by means of identifying affordable features suitable for SMEs' decisionmaking. The affordable features that have been mentioned in the literature need to be tested. For this reason, the philosophy selected for the research was positivism as it emphasises on the practical application of features related to IBM Cognos and testing them in human experiences related with the decision-making needs of SMEs. According to Blanco, García-Peñalvo and Sein-Echaluce (2013), positivism leads to the assessment and evaluation of varied beliefs and theories. Research with a deductive approach, explain Saunders, Lewis and Thornhill (2016), develops a conceptual framework based on existing theory. A deductive approach was suitable; this study used a signification framework (outlined further below) and theory of business decision-making for investigating and finding out the affordability of IBM Cognos features.

The research strategy was a survey to gather data related to the affordable features of IBM Cognos and SMEs' decisionmaking needs. According to Creswell and Creswell (2017), survey strategy leads to gathering accurate data related to the topic of the study. Survey strategy was appropriate for a quantitative study. The three major types of research designs are quantitative, qualitative and mixed methodology (Mertler \& Reinhart 2016). For this study, the variables of the study were quantified and measured by statistical tools. Quantitative research design was necessary to retain the objectivity of the study and deduce concise results out of the available information. Thus, to find the significance of the fundamental features of IBM Cognos BI tools, a quantitative design was the most suitable choice.

Primary data-collection method was the way of gathering data using a structured questionnaire, a quantitative monomethod. Saunders et al. (2016:167) describe using a 'single data collection technique, such as a questionnaire, and corresponding quantitative analytical procedure' aligned to positivism. The online questionnaire link was shared with SMEs; a non-probability convenience sampling technique was used to select the research participants.

The population comprised SMEs in the Gauteng province of South Africa which was further narrowed down to all the SMEs listed in the service and retail industry (Brabys.com 2018). The sample size was 200 respondents out of which 80 were BI consultants from consultancy firms, 60 SME BI developers and 60 SME managers. The questionnaire was based on the signification framework's five categories of significance:

- Presume: What is the presumed relevance of a BI tool such as IBM Cognos? 


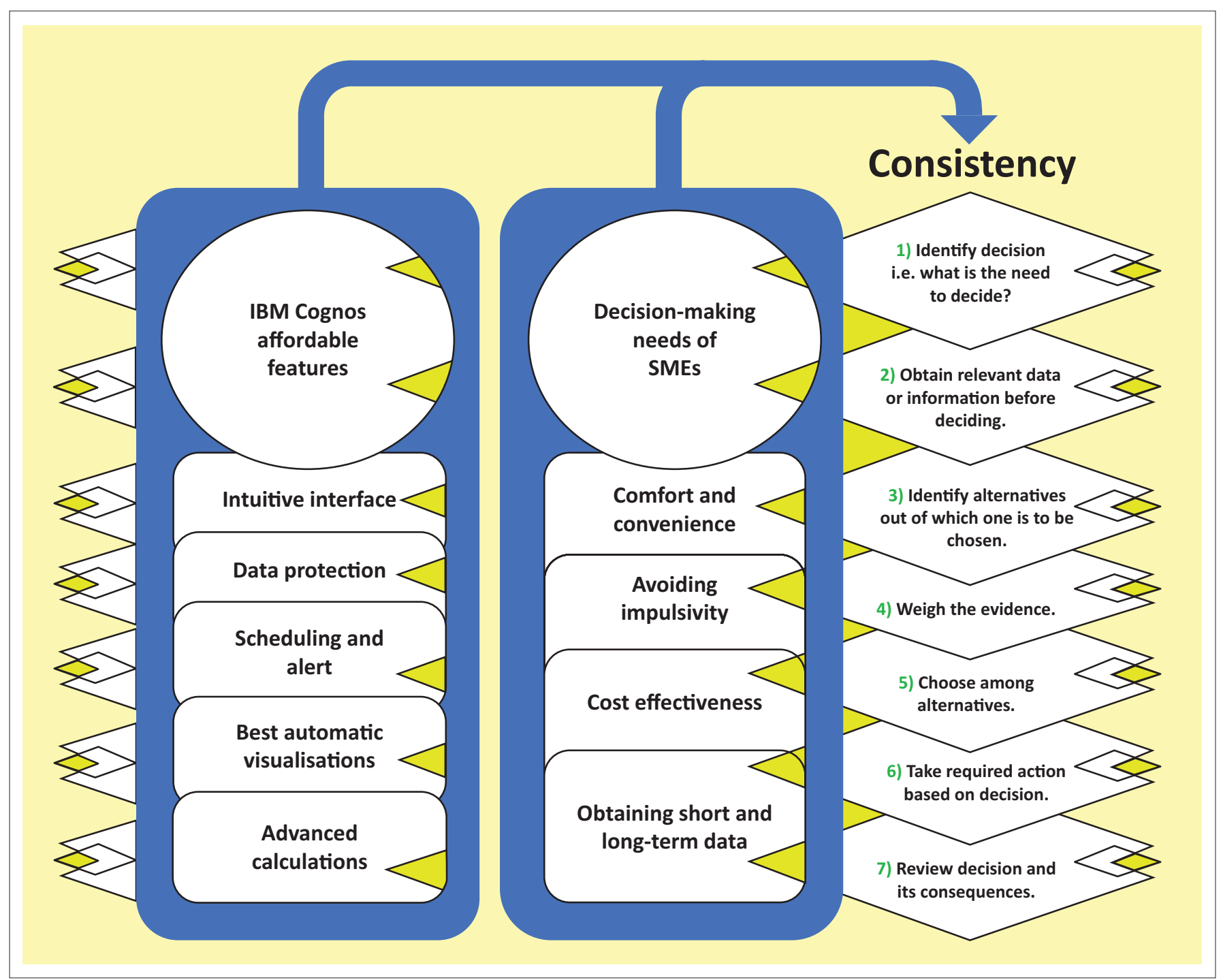

SME, small- and medium-sized enterprises.

FIGURE 1: The conceptual framework.

- Predict: How would an SME's business decision-making ability change as a consequence of IBM Cognos features?

- Prize: What are the key features of IBM Cognos?

- Perceive: How do SME users of BI tools perceive IBM Cognos features?

- Produce: What do IBM Cognos features produce which is of value to SMEs?

The delimitation of study was the affordability of the fundamental features of IBM Cognos leading to affect the decision-making needs of SMEs in Gauteng, South Africa. The scope was not extended to an investigation of adoption behaviour. The scope was the affordability of BI tools in serving SMEs' decision-making needs based on research results and analysis.

\section{Ethical considerations}

Ethical clearance was obtained from the College of Business and Economics, Research Ethics Committee, University of Johannesburg, ethical clearance code IKM2018_033_ KHUMALO_DUPLESSIS_MAGOMA.

\section{Results and analysis}

The data were collected from BI consultants, SME managers and SME BI developers. The response rate was 100\%. A total of 25 questions were asked from the participants and three variables were used: SME's decision-making needs, IBM Cognos BI tool features and BI tool affordability. The researchers explored and investigated the variables in order to assess which of the fundamental features of IBM Cognos were affordable to address the decision-making needs of SMEs. To begin with, the signification framework research setting was introduced by means of statements about the extent to which participants prized particular IBM Cognos features above other features:

- View data easily and conveniently.

- Modify data easily and conveniently.

- Modified data help the organisation to address the queries of users.

- Access to relevant information.

- Data encryption with the help of codes or passwords.

- Scheduling and alerts allow keeping the information up to date. 
- Being made aware of any cost overruns or overbearing expenses.

- Data visualisation helps to take better decisions in the organisation.

- Scheduling enables the organisation to take better business decisions.

- Scheduling enables the organisation to take better financial decisions.

- Alerts enable the organisation to take better business decisions.

- Alerts enable the organisation to take better financial decisions.

Ten questions were asked which were related to IBM Cognos tool features, seven questions related to affordability and eight were asked related to decision-making needs of SMEs. This article presents the key findings from some, not all, of the questions. For this article, the first question determined to which extent respondents presumed statements about IBM Cognos features to be true (Figure 2).

It was discovered that respondents presumed that IBM Cognos aids financial decision-making and alerts SMEs with cost overruns (Figure 2). In terms of financial decisions, many respondents answered most probable $(63.27 \%)$ or likely (33.67\%); whereas few respondents answered unlikely $(1.02 \%)$ or totally improbable $(1.01 \%)$. In terms of cost overruns, many respondents answered most probable $(61.62 \%)$ or likely $(36.36 \%)$; whereas few respondents answered unlikely $(1.01 \%)$ or totally improbable $(1.01 \%)$. The features of financial decisions aid and avoiding overbearing expenses were most presumed in the signification framework.

Respondents also identified the scheduling feature, data visualisation, information access, data encryption, scheduling and alerts, modified data, view and modify data and databases as the features that best characterised IBM Cognos.

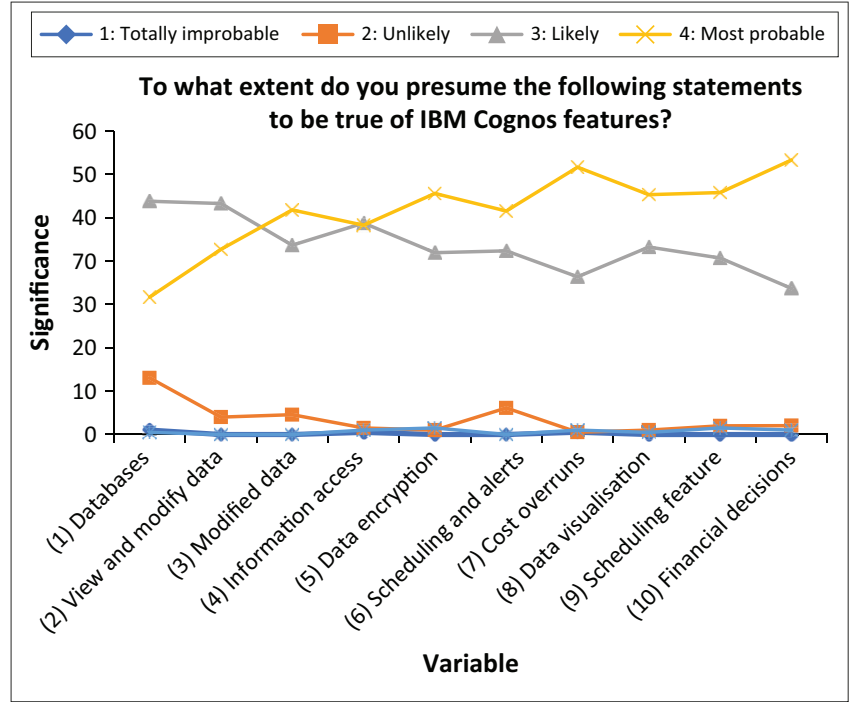

FIGURE 2: Presumed IBM Cognos features.
The next set of questions was related to whether respondents agreed with statements related to the fundamental features of IBM Cognos (Figure 3). These statements made use of signification framework terminology, such as produce and predict, in order to identify features of IBM Cognos that should be included in the SMEs' decision-making toolkit based on SMEs' decision-making needs.

Figure 3 illustrates the fundamental features of IBM Cognos that are of low cost and high quality $(61.5 \%$ totally agree and $37.5 \%$ somewhat agree) and its low-cost feature $(58.59 \%$ totally agree and $37.37 \%$ somewhat agree).

Furthermore, the features for data visualisation, affordability and system implementation should be made part of SMEs' decision-making toolkit. Features linked to affordability, cost effective, low cost and affordability as a factor are clearly a priority for SMEs as indicated by most of the respondents in total agreement of statements linked to the features of IBM Cognos.

Next, Figure 4 showcases the statements in relation to the extent to which respondents prized certain IBM Cognos features above certain other features.

In Figure 4, the results show that the most prized feature of IBM Cognos was data visualisation, as indicated by $66.50 \%$ of respondents. Statements in relation to consistency were the features for scheduling and decision-making process: financial decisions prized by $58.46 \%$ of respondents, better decisions by $57.87 \%$ of respondents, business decisions by $54.31 \%$ of respondents and scheduling by $56.5 \%$ of respondents. Overall, the results show that modifying data, view data easily, access to information, data encryption, scheduling and alerts, data visualisation and making better business decisions and financial decisions are considered highly important. A comparison of the trend lines in Figures 2 and 4 illustrates, for example, that presumed significance of

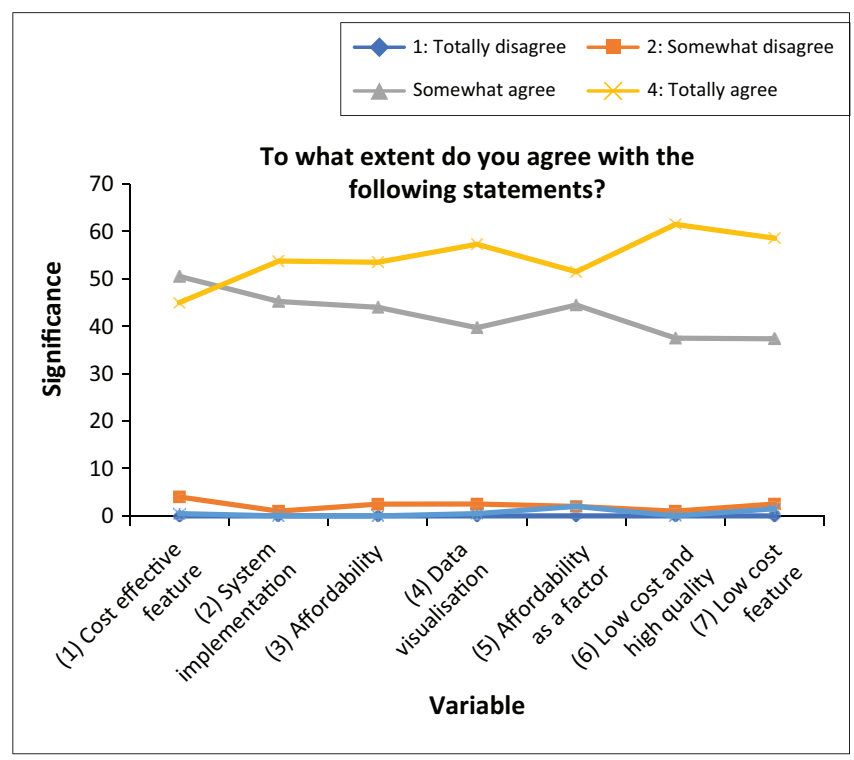

FIGURE 3: Fundamental IBM Cognos features. 
data visualisation is surpassed by the prized significance of the data visualisation feature. As for the features associated with decision-making, financial decision-making was presumed by $63.27 \%$ of respondents, whereas $58.46 \%$ of respondents prized financial decision-making. In Figure 4, compared to Figure 3, 51\% of respondents prized the features of IBM Cognos assisting them in avoiding cost overruns, and in Figure 3,61.6\% of respondents presumed the feature of avoiding cost overruns. The respondents also prized data encryption, access to information, user queries, modified data and data accessibility features of IBM Cognos.

Next, Figure 5 presents results for the identification of IBM Cognos features as perceived by the respondents.

Figure 5 identifies the four most perceived features of IBM Cognos as perceived by SMEs, namely scheduling and alerts $(62.76 \%)$, data encryption $(62.5 \%)$, scheduling $(62.5 \%)$ and financial decision-making (62.3\%). Respondents always perceived these features; modified data (56.48\%), view and modify data $(52.26 \%)$, access to information $(55.76 \%)$, cost overruns $(56.78 \%)$ and data visualisation $(57.7 \%)$. In Figure 5, the four most perceived features coincide with presumed and prized features; for example, data encryption is highly important (Figure 4) and data encryption is most probable (Figure 2), which means the results of this study identified the fundamental features of relevance to the decision-making needs of SMEs, as discussed next.

\section{Discussion}

Three themes emerged from the data, namely the SME business decision-making context, affordability and the fundamental features of IBM Cognos (Magoma 2018). All the questions asked from the respondents were associated with IBM Cognos' features and how they are effective for decisionmaking in SMEs. The results indicate that BI consultants, SME managers and BI developers representing SMEs are aware of the BI tool features of IBM Cognos. Respondents identified IBM Cognos tools in support of financial decisions,

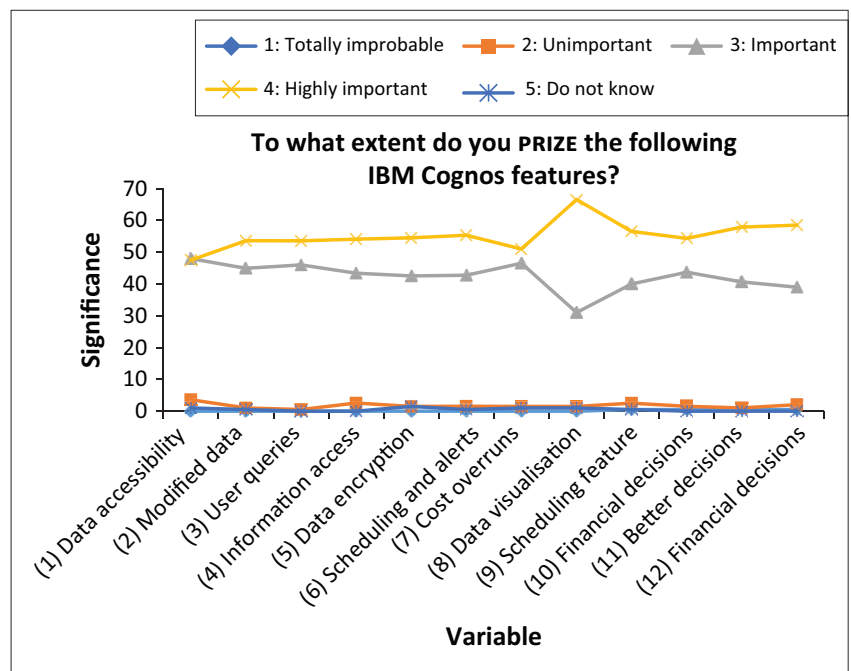

FIGURE 4: Prized IBM Cognos features. modifying data, data encryption, scheduling and alerts and data visualisation as the features through which BI plays a significant role in SMEs' decision-making. Respondents mostly presumed the features in support of financial decisionmaking and for alerting users to avoid cost overruns, whereas the most prized feature was data visualisation. Although the BI tool allowed users to modify data, affordability was more important for SMEs. Small- and medium-sized enterprises benefit from BI tools especially in terms of avoiding cost overruns. The data obtained from respondents support the literature review findings in that BI would be helpful in terms of managing the data more systematically if it was affordable. Features that are cost wise and implemented easily enable SMEs to report easily and quickly.

It was identified from the literature review that one of the major concerns for SMEs is management of data as a precondition of staying competitive and sustainable (Ghazanfari et al. 2011). The analysis of results of this study identified those features of IBM Cognos that present various presumed, prized and perceived features for data visualisation, access to information and data encryption, which Cascetta et al. (2015) describe as data management features that assist businesses in taking adequate decisions. Effective data management indicates that businesses are able to improve their overall efficiency that allows them to attain an edge and advantage over their competitors (Roitzsch et al. 2012).

It has been understood from the literature review that storing and managing data is important for SMEs, and this is one of the major reasons how SMEs are able to sustain their position in the market. The results of the study have showed that $\mathrm{BI}$ tools lead to resolve various issues for the companies. More than $90 \%$ of the total respondents agreed to the statement that by using BI tools, SMEs were making better decisions. It has further been observed from the results that more than $90 \%$ of the respondents agreed that BI tools guard against cost overruns.

Even though the significance of BI tools has been acknowledged, BI tools would have had more significance when features were affordable. The literature review identified

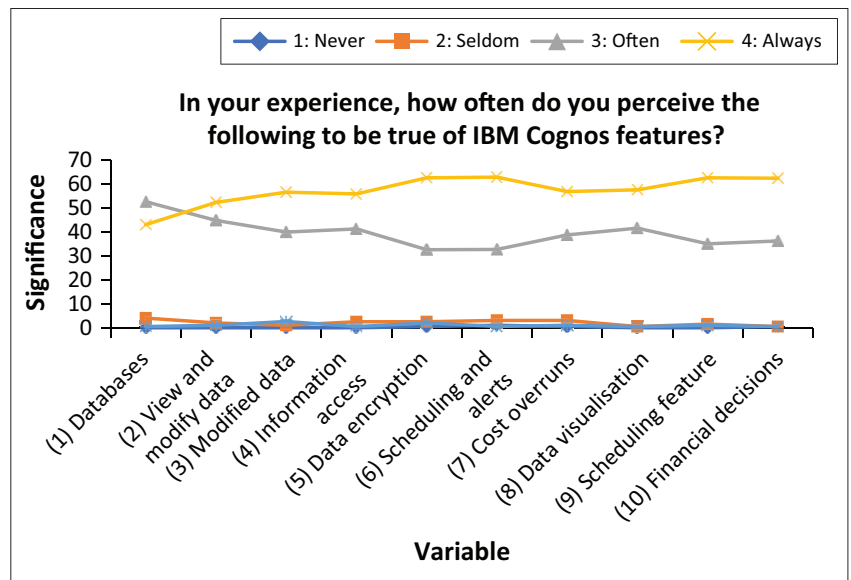

FIGURE 5: Identification of IBM Cognos features as perceived by respondents. 
IBM Cognos as an effective tool that has been used by large organisations to manage information more adequately (Blanco et al. 2013). Pertaining to small organisations, the results of this study show that SMEs' effective decision-making depends on access to cost-effective features of BI tools. Findings indicate that IBM Cognos could result in being an effective tool for organisations through which they may lead to resolve datamanagement issues. With the implementation of BI tools, SMEs would be able to enhance their overall performance and lead towards better sustainability in the market.

In summary, the significance of affordable key features of BI tools in the context of SME business decision-making includes consistency and comfort, intuitive interface, avoiding impulsivity, cost effectiveness, availability of information, best programmed visualisations and reporting quickly and easily.

\section{Conclusion and recommendation}

The unit of analysis was affordability as a feature of IBM Cognos that assists SMEs in the process of decision-making. The main discovery from the literature review was that consistency is key to SMEs' decision-making and SMEs require $\mathrm{BI}$ tool intuitive interface. The interface relates to convenience and ease of authoring content, designing, building and securing reports to the SME, which helps in improving overall decision-making. Key findings from the primary data were the presumed, prized and perceived value indicators, which include features linked to avoiding cost overruns, data visualisation, data encryption, access to information, user queries, modified data, data accessibility and financial decision-making features.

This study compared the presumed, prized and perceived value of the fundamental features of IBM Cognos using a signification framework and presents an alternative view of the affordability of BI tools. Affordability does not necessarily refer to low cost; instead, consistent decision-making is valued by SMEs; therefore, affordable BI tool features are those features that prelude consistency. If it does not bring about consistency, it is not affordable.

Affordable BI tool features, in combination with a correct understanding of the decision-making needs of SMEs, produce consistency. Small- and medium-sized enterprise managers will benefit from BI tools because it presents a consistent way of making choices based on identifying the needs, gathering information and assessing alternatives.

It is recommended that SMEs acquire affordable BI tools that fulfil their decision-making needs. Small- and medium-sized enterprises must investigate the signification framework method to identify the presumed, predicted, perceived, prized and produced decision-making value of BI tools before acquiring BI tools. Small-and medium-sized enterprises and BI software developers must discuss the mediating role of affordable BI tool features for consistent decision-making.

\section{Value of study}

This study is important for SMEs to enhance decision-making by using BI tools. The affordability of fundamental BI tool features that are suitable for SMEs is an important issue. The study identified affordable IBM Cognos BI tool features to assist SMEs' consistent decision-making (Magoma 2018).

\section{Future studies}

Qualitative research design would allow the study to analyse BI professionals' responses based on their knowledge and skills regarding BI tools. Qualitative data analysis would be suitable to test this study's definition of 'affordable' based on the signification framework indicators of the value of BI tools for SMEs.

\section{Acknowledgements Competing interests}

The authors declare that they have no financial or personal relationships that may have inappropriately influenced them in writing this article.

\section{Authors' contributions}

T.M., S.K and T.d.P. contributed equally to this work.

\section{Funding information}

This research received no specific grant from any funding agency in the public, commercial or not-for-profit sectors.

\section{Data availability}

The data that support the findings of this study are available from any of the three authors; the corresponding author is T.d.P.; data can be shared upon reasonable request.

\section{Disclaimer}

The views and opinions expressed in this article are those of the authors and do not necessarily reflect the official policy or position of any affiliated agency of the authors.

\section{References}

Adkison, D., 2013, IBM Cognos business intelligence, Packt Publishing, Birmingham.

Antons, D. \& Piller, F.T., 2015, 'Opening the black box of "not invented here": Attitudes, decision biases, and behavioural consequences', Academy of Management Perspectives 29(2), 193-217. https://doi.org/10.5465/amp.2013.0091

Ates, A., Garengo, P., Cocca, P. \& Bititci, U., 2013, 'The development of SME managerial practice for effective performance management', Journal of Small Business and Enterprise Development 20(1), 28-54. https://doi.org/10.1108/14626001 311298402

Batt, S., Grealis, T., Harmon, O. \& Tomolonis, P., 2020, 'Learning Tableau: A data visualisation tool', Journal of Economic Education 51(3/4), 317-328. https://doi.or $\mathrm{g} / 10.1080 / 00220485.2020 .1804503$

Becerra-Godínez, J.A., Serralde-Coloapa, J.L., Ulloa-Márquez, M.S., Gordillo-Mejía, A. \& Acosta-Gonzaga, E., 2020, 'Identifying the main factors involved in business intelligence implementation in SMEs', Bulletin of Electrical Engineering and Informatics 9(1), 304-310. https://doi.org/10.11591/eei.v9i1.1459

Blanco, Á.F., García-Peñalvo, F.J. \& Sein-Echaluce, M., 2013, 'A methodology proposal for developing adaptive MOOC', Proceedings of the first International conference on technological ecosystem for enhancing multiculturality, pp. 553-558, Association for Computing Machinery (ACM), New York, NY. 
BMI Research Technology Report, 2018, South Africa information technology report Q3, Canary Wharf, London.

Brabys.com, 2018, Online map and business search directory for South Africa, viewed 12 December 2018, from http://www.brabys.com.

Canes, M., 2009, 'Business intelligence for the SME', CA Magazine 142(7), 46-48.

Cascetta, E., Carteni, A., Pagliara, F. \& Montanino, M., 2015, 'A new look at planning and designing transportation systems: A decision-making model based on cognitive rationality, stakeholder engagement, and quantitative methods' Transport Policy 38(C), 27-39. https://doi.org/10.1016/j.tranpol.2014.11.005

Chii, Y.Y., Xue, F., Low, W.W., Yoon, J.H. \& Gold, S., 2016, 'A big data approach for memory quality management', Region 10 conference (TENCON), Singapore, November 22-25, 2016, pp. 2448-2452.

Chng, D.H.M., Shih, E., Rodgers, M.S. \& Song, X.B., 2015, 'Managers' marketing strategy decision-making during performance decline and the moderating influence of incentive pay', Journal of the Academy of Marketing Science 43(5) influence of incentive pay', Journal of the Academy of
$629-647$. https://doi.org/10.1007/s11747-014-0401-x

Cooney, M., 2020, 'IBM set to spin-off managed service business to focus on hybrid cloud', Computerworld, viewed 22 December 2020, from https://0-web-bebscohost-com.ujlink.uj.ac.za/ehost/.

Creswell, J.W. \& Creswell, J.D., 2017, Research design: Qualitative, quantitative, and mixed methods approach, Sage, Newcastle.

Davis, G.A. \& Woratschek, C.R., 2015, 'Evaluating business intelligence/business analytics software for use in the information systems curriculum', Information Systems Education Journal 13(1), 23.

Eden, C. \& Ackermann, F., 2013, Making strategy: The journey of strategic management, Sage, Newcastle.

Ehlers, T., Lazenby, K., Cronjé, S., Maritz, R. \& Letsholo, R., 2019, Strategic management Southern African concepts and cases, Van Schaik Publishers, Pretoria.

European Commission, 2019, 'Supporting specialised skills development: Big data, Internet of things and cybersecurity for SMEs, Interim report: EASME/ COSME/2017/007, viewed 22 December 2020, from https://www.digitalsme.eu/ digital/uploads/March-2019_Skills-for-SMEs_Interim_Report_final-version.pdf.

Fan, S., Lau, R.Y. \& Zhao, J.L., 2015, 'Demystifying big data analytics for business intelligence through the lens of marketing mix', Big Data Research 2(1), 28-32. https://doi.org/10.1016/j.bdr.2015.02.006

Gangadharan, G.R. \& Swami, S.N., 2004, 'Business intelligence systems: Design and implementation strategies', 26th International conference on information technology interfaces IEEE, Cavtat, Croatia, June 07-10, 2004, pp. 139-144.

Ghazanfari, M., Jafari, M. \& Rouhani, S., 2011, 'A tool to evaluate the business intelligence of enterprise systems', Scientia Iranica 18(6), 1579-1590. https://doi. org/10.1016/j.scient.2011.11.011

Gurjar, Y.S. \& Rathore, V.S., 2013, 'Cloud business intelligence - Is what business need today', International Journal of Recent Technology and Engineering 1(6), 81-86.

Horakova, M. \& Skalska, H., 2013, 'Business intelligence and implementation in a small enterprise', Journal of Systems Integration 4(2), 50-61.

International Business Machines (IBM), 2010, IBM System storage report, Redbooks, Poughkeepsie, NY.

International Business Machines (IBM), 2012, IBM Power 720 and 740 technical overview and introduction report, Redbooks, Poughkeepsie, NY.

International Business Machines (IBM), 2013, IModernise your application infrastructure, Redbooks, Poughkeepsie, NY.

Kim, J., Ostrowski, D.A., Yamaguchi, H. \& Sheu, P.C.Y., 2013, 'Semantic computing and business intelligence', International Journal of Semantic Computing 7(1), 87-117. https://doi.org/10.1142/S1793351X13500013

Kimball, R., Ross, M., Becker, B., Mundy, J. \& Thornthwaite, W., 2015, The Kimball group reader: Relentlessly practical tools for data warehousing and business intelligence re-mastered collection, John Wiley \& Sons, Hoboken, NJ.

Kousalya, G., Balakrishnan, P. \& Raj, C.P. (eds.), 2017, Automated workflow scheduling in self-adaptive clouds: Concepts, algorithms and methods, Springer, Cham.

Ladzani, W.M. \& Van Vuuren, J.J., 2002, 'Entrepreneurship training for emerging SMEs in South Africa', Journal of Small Business Management 40(2), 154-161. https:// doi.org/10.1111/1540-627X.00047

Larson, D. \& Chang, V., 2016, 'A review and future direction of agile, business intelligence, analytics, and data science', International Journal of Information Management 36(5), 700-710. https://doi.org/10.1016/j.jijinfomgt.2016.04.013

Lim, E.P., Chen, H. \& Chen, G., 2013, 'Business intelligence and analytics: Research directions', ACM Transactions on Management Information Systems 3(4), 17 https://doi.org/10.1145/2407740.2407741
Llave, M.R., Hustad, E. \& Olsen, D.H., 2018, 'Creating value from business intelligence and analytics in SMEs: Insights from experts', 24th Americas conference on information systems, New Orleans, LA, United States of America, January, 2018, pp. 194-205.

Loshin, D., 2012, Business intelligence: The savvy manager's guide, Newnes, San Francisco, CA

Magoma, T.C., 2020, 'Signification framework of a business intelligence tool for SME business decision-making', M.Com minor dissertation, Department of Information and Knowledge Management, University of Johannesburg, Johannesburg.

Mathu, K.M., 2019, 'The information technology role in supplier-customer information-sharing in the supply chain management of South African small and medium-sized enterprises', South African Journal of Economic and Management medium-sized enterprises', South African Journal of Economic and
Sciences 22(1), 1-8. https://doi.org/10.4102/sajems.v22i1.2256

Mayeng, N. \& Van Vuuren, J., 2017, 'The benefits of social media as digital marketing tool by SMMEs', Proceedings of the 1st International conference on entrepreneurship development (ICED), April 5-7, 2017, Unit for Enterprise Studies, Faculty of Management Sciences, Central University of Technology, Free State.

Merriam-Webster Dictionary, 2020, Affordability, viewed 22 December 2020, from https://www.merriam-webster.com/dictionary/affordability.

Mertler, C.A. \& Reinhart, R.V., 2016, Advanced and multivariate statistical methods: Practical application and interpretation, Routledge, Abingdon.

Nogués, A. \& Valladares, J., 2017, Business intelligence tools for small companies: A guide to free and low cost solutions, Springer, New York, NY.

OED (Oxford English Dictionary), 2020a, Affordable, viewed 22 December 2020, from https://0-www-oed-com.ujlink.uj.ac.za/view/Entry/3484.

OED (Oxford English Dictionary), 2020b, Significance, viewed 22 December 2020, from https://0-www-oed-com.ujlink.uj.ac.za/view/Entry/179566.

Oztemel, E. \& Gursev, S., 2020, 'Literature review of Industry 4.0 and related technologies', Journal of Intelligent Manufacturing 31, 127-182. https://doi. org/10.1007/s10845-018-1433-8

Pettigrew, A.M., 2009, The politics of organisational decision-making, Digital reprint, Routledge, Oxon.

Republic of South Africa (RSA), The Presidency, 2003, Act No. 26 of 2003, National Small Business Amendment Act, Government Gazette No. 25763, November 26, Pretoria.

Richardson, J., Sallam, R., Schlegel, K., Kronz, A. \& Sun, J., 2020, Gartner magic quadrant for analytics and business intelligence platforms, viewed 22 Decembe 2020, from https://www.gartner.com/en/documents/3980852/magic-quadrantfor-analytics-and-business-intelligence-p.

Roitzsch, K., Hacker, W., Pietrzyk, U. \& Debitz, U., 2012, 'How do German SMEs cope with the increasing need for flexibility?', Advances in Decision Sciences, 2012, 1-13. https://doi.org/10.1155/2012/569076

Rusaneanu, A., 2013, 'Comparative analysis of the main business intelligence solutions', Informatica Economica 17(2), 148-156. https://doi.org/10.12948/ issn14531305/17.2.2013.12

Saunders, M., Lewis, P. \& Thornhill, A., 2016, Research methods for business students, 7 th edn., Pearson Education, Harlow.

Smith, W.K., 2014, 'Dynamic decision-making: A model of senior leaders managing strategic paradoxes', Academy of Management Journal 57(6), 1592-1623. https:// doi.org/10.5465/amj.2011.0932

Steinerowska-Streb, I. \& Steiner, A., 2014, 'An analysis of external finance availability on SMEs' decision-making: A case study of the emerging market of Poland', Thunderbird International Business Review 56(4), 373-386. https://doi. org/10.1002/tie.21627

Thamir, A. \& Poulis, E., 2015, 'Business intelligence capabilities and implementation strategies', International Journal of Global Business 8(1), 34-55.

Turban, E., King, D., Sharda, R. \& Delen, D., 2013, Business intelligence: A managerial perspective on analytics, Prentice Hall, New York, NY.

Turi, A.N., 2020, 'Digital economy and the information society', in A.N. Turi (ed.), Technologies for modern digital entrepreneurship, pp. 1-41, Apress, Berkeley, CA.

Wagner, R. \& Paton, R.A., 2014, 'Strategic toolkits practice and performance: A German SME perspective', VINE: The Journal of Information and Knowledge Management Systems 44(4), 470-495. https://doi.org/10.1108/VINE-03-2014-0019

Wu, X., Zhu, X., Wu, G.Q. \& Ding, W., 2014, 'Data mining with big data', IEEE Transactions on Knowledge and Data Engineering 26(1), 97-107. https://doi. org/10.1109/TKDE.2013.109 